\title{
Application of chiral perturbation theory to $2+1$ flavor lattice QCD with $O(a)$-improved Wilson quarks
}

\author{
PACS-CS Collaboration: D. Kadoh ${ }^{*} \dagger$, S. Aoki ${ }^{b, c}$, N. Ishii ${ }^{a}$, K.-I. Ishikawa ${ }^{d}$, \\ N. Ishizuka ${ }^{a, b}$, T. Izubuchi ${ }^{c, e}$, K. Kanaya ${ }^{b}$, Y. Kuramashi ${ }^{a, b}$, Y. Namekawa ${ }^{a}$, \\ M. Okawa ${ }^{d}$, K. Sasaki $^{a}$, Y. Taniguchi ${ }^{a, b}$, A. Ukawa ${ }^{a, b}$, N. Ukita ${ }^{a}$ T. Yoshié $^{a, b}$ \\ ${ }^{a}$ Center for Computational Sciences, University of Tsukuba, Tsukuba, Ibaraki 305-8577, Japan \\ ${ }^{b}$ Graduate School of Pure and Applied Sciences, University of Tsukuba, Tsukuba, Ibaraki \\ 305-8571, Japan \\ ${ }^{c}$ Riken BNL Research Center, Brookhaven National Laboratory, Upton, New York 11973, USA \\ ${ }^{d}$ Department of Physics, Hiroshima University, Higashi-Hiroshima, Hiroshima 739-8526, Japan \\ ${ }^{e}$ Institute for Theoretical Physics, Kanazawa University, Kanazawa, Ishikawa 920-1192, Japan
}

\begin{abstract}
We apply chiral perturbation theory to the pseudoscalar meson mass and decay constant data obtained in the PACS-CS Project toward 2+1 flavor lattice QCD simulations with the $O(a)$-improved Wilson quarks. We examine the existence of chiral logarithms in the quark mass range from $m_{\mathrm{ud}}=47 \mathrm{MeV}$ down to $6 \mathrm{MeV}$ on a $(2.8 \mathrm{fm})^{3}$ box with the lattice spacing $a=0.09 \mathrm{fm}$. Several low energy constants are determined. We also discuss the magnitude of finite size effects based on chiral perturbation theory.
\end{abstract}

The XXV International Symposium on Lattice Field Theory

July 30-4 August 2007

Regensburg, Germany

\footnotetext{
${ }^{*}$ Speaker.

†E-mail: kadoh@ccs.tsukuba.ac.jp
} 


\section{Introduction}

The PACS-CS Collaboration has been pushing $N_{F}=2+1$ full QCD simulations toward the physical point with the nonperturbatively $O(a)$-improved Wilson quark action and the Iwasaki gauge action $[1,2,3]$. As presented in a separate report [4] in detail, sizable amount of hadron spectrum data has been collected at $\beta=1.9$ on a $32^{3} \times 64$ lattice down to the pion mass $m_{\pi}=210 \mathrm{MeV}$ using the domain-decomposed Hybrid Monte Carlo (DDHMC) algorithm [5] on the PACS-CS computer. In this report we examine the chiral behavior of the pseudoscalar meson masses and decay constants in comparison with the prediction of chiral perturbation theory (ChPT). In particular, we focus on the three points: (i) signals for chiral logarithms, (ii) determination of low energy constants in the chiral lagrangian, (iii) determination of the physical point with the ChPT fit.

\section{Simulation parameters}

We employ the $O(a)$-improved Wilson quark action with a nonperturbative improvement coefficient $c_{\mathrm{SW}}=1.715[6]$ and the Iwasaki gauge action at $\beta=1.9$ on a $32^{3} \times 64$ lattice. In Table 1 we summarize the hopping parameters $\left(\kappa_{\mathrm{ud}}, \kappa_{\mathrm{s}}\right)$ and the results for the pion masses and the unrenormalized quark masses. The latter is defined through the axial vector Ward-Takahashi identity (AWI) by

$$
a m_{q}^{\mathrm{AWI}}=\lim _{t \rightarrow \infty} \frac{\left\langle\nabla_{4} A_{4}^{\mathrm{imp}}(t) P(0)\right\rangle}{2\langle P(t) P(0)\rangle}
$$

where $P$ is the pseudoscalar density and $A_{4}^{\mathrm{imp}}$ is the nonperturbatively $O(a)$-improved axial vector current[7].

For later use we also define the renormalized quark mass and the pseudoscalar meson decay constant in the continuum $\overline{\mathrm{MS}}$ scheme as follows:

$$
\begin{aligned}
m_{q}^{\overline{\mathrm{MS}}} & =\frac{Z_{A}\left(1+b_{A} \frac{m^{\mathrm{AWI}}}{u_{0}}\right)}{Z_{P}\left(1+b_{P} \frac{m^{\mathrm{AWI}}}{u_{0}}\right)} m_{q}^{\mathrm{AWI}}, \\
f_{\mathrm{PS}} & =2 \kappa u_{0} Z_{A}\left(1+b_{A} \frac{m_{q}^{\mathrm{AWI}}}{u_{0}}\right) \frac{C_{A}^{s}}{C_{P}^{s}} \sqrt{\frac{2 C_{P}^{l}}{m_{P S}}} .
\end{aligned}
$$

Here $C_{A, P}^{s}$ are the amplitudes extracted from the correlation functions $\left\langle A_{4}^{\mathrm{imp}}(t) P(0)\right\rangle$ and $\langle P(t) P(0)\rangle$ with the exponentially smeared source and the local sink, while $C_{P}^{l}$ is from $\langle P(t) P(0)\rangle$ with the local source and the local sink. The renormalization factors $Z_{A, P}$ and the improvement coefficients $b_{A, P}$ are evaluated perturbatively up to one-loop level[8,9]with the tadpole improvement.

\section{Results}

\subsection{Comparison with the previous CP-PACS/JLQCD results}

We first compare our results with the previous CP-PACS/JLQCD results, both of which are obtained with the same quark and gauge actions at $\beta=1.9$ but on different lattice sizes: $32^{3} \times 64$ for 


\begin{tabular}{cccccc}
\hline$\kappa_{\mathrm{ud}}$ & $\kappa_{\mathrm{s}}$ & $a m_{\pi}$ & $a m_{\mathrm{ud}}^{\mathrm{AWI}}$ & $a m_{\mathrm{s}}^{\mathrm{AWI}}$ & MD time \\
\hline \hline 0.13700 & 0.13640 & $0.32196(62)$ & $0.02800(20)$ & $0.04295(30)$ & 2000 \\
0.13727 & 0.13640 & $0.26190(66)$ & $0.01895(13)$ & $0.04061(18)$ & 2000 \\
0.13754 & 0.13640 & $0.18998(56)$ & $0.01020(11)$ & $0.03876(18)$ & 2500 \\
0.13770 & 0.13640 & $0.13591(88)$ & $0.00521(9)$ & $0.03767(10)$ & 2000 \\
0.13781 & 0.13640 & $0.08989(291)$ & $0.00227(16)$ & $0.03716(20)$ & 350 \\
\hline 0.13754 & 0.13660 & $0.17934(78)$ & $0.00908(7)$ & $0.03257(17)$ & 900 \\
\hline
\end{tabular}

Table 1: Hopping parameters for the up-down and the strange quarks together with the results of $a m_{\pi}$, $a m_{\mathrm{ud}}^{\mathrm{AWI}}$ and $a m_{\mathrm{s}}^{\mathrm{AWI}}$. MD time is the number of trajectories multiplied by the trajectory length.

the former and $20^{3} \times 40$ for the latter. For the hopping parameters only the combination $\left(\kappa_{\mathrm{ud}}, \kappa_{\mathrm{s}}\right)=$ $(0.13700,0.13640)$ is in common, which is the heaviest case in the PACS-CS results whereas it is the lightest one in the CP-PACS/JLQCD results.

In Fig. 1 we plot $\left(a m_{\pi}\right)^{2} /\left(a m_{\mathrm{ud}}^{\mathrm{AWI}}\right)$ and $f_{K} / f_{\pi}$ as a function of $a m_{\mathrm{ud}}^{\mathrm{AWI}}$ with $\kappa_{\mathrm{s}}$ fixed at 0.13640 . The PACS-CS and the CP-PACS/JLQCD results are denoted by the black and the red symbols, respectively. The two sets of data together show a smooth behavior as a function of $a m_{\mathrm{ud}}^{\mathrm{AWI}}$, and at $\kappa_{\mathrm{ud}}=0.13700\left(a m_{\mathrm{ud}}^{\mathrm{AWI}}=0.028\right)$ they show good consistency.

An important observation is that while the CP-PACS/JLQCD results show an almost linear quark mass dependence both for $\left(a m_{\pi}\right)^{2} /\left(a m_{\mathrm{ud}}^{\mathrm{AWI}}\right)$ and $f_{K} / f_{\pi}$, we find a clear curvature for the PACS-CS results, which is a characteristic feature of the ChPT prediction in the small quark mass region. This curvature drives the PACS-CS results for $f_{K} / f_{\pi}$ close to the experimental value toward the physical point.
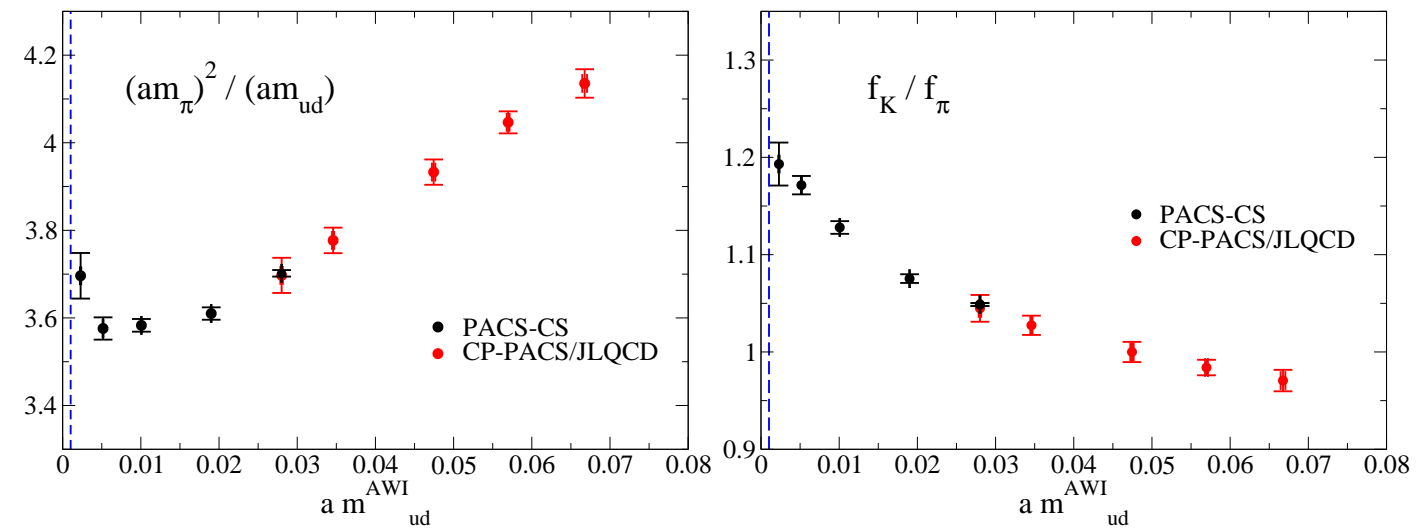

Figure 1: Comparison of the PACS-CS (black) and the CP-PACS/JLQCD (red) results for $\left(a m_{\pi}\right)^{2} /\left(a m_{\mathrm{ud}}^{\mathrm{AWI}}\right)$ (left) and $f_{K} / f_{\pi}$ (right). $\kappa_{\mathrm{s}}$ is fixed at 0.13640 .

\subsection{Chiral fit formulae}

In ChPT, the one-loop expressions for the pseudoscalar meson masses and the decay constants are given by[10]

$$
m_{\pi}^{2}=2 \hat{m} B_{0}\left\{1+\mu_{\pi}-\frac{1}{3} \mu_{\eta}+\frac{B_{0}}{f_{0}^{2}}\left(16 \hat{m}\left(2 L_{8}-L_{5}\right)+16\left(2 \hat{m}+m_{\mathrm{s}}\right)\left(2 L_{6}-L_{4}\right)\right)\right\}
$$




$$
\begin{aligned}
m_{K}^{2} & =\left(\hat{m}+m_{\mathrm{s}}\right) B_{0}\left\{1+\frac{2}{3} \mu_{\eta}+\frac{B_{0}}{f_{0}^{2}}\left(8\left(\hat{m}+m_{\mathrm{s}}\right)\left(2 L_{8}-L_{5}\right)+16\left(2 \hat{m}+m_{\mathrm{s}}\right)\left(2 L_{6}-L_{4}\right)\right)\right\}, \\
f_{\pi} & =f_{0}\left\{1-2 \mu_{\pi}-\mu_{K}+\frac{B_{0}}{f_{0}^{2}}\left(8 \hat{m} L_{5}+8\left(2 \hat{m}+m_{\mathrm{s}}\right) L_{4}\right)\right\} \\
f_{K} & =f_{0}\left\{1-\frac{3}{4} \mu_{\pi}-\frac{3}{2} \mu_{K}-\frac{3}{4} \mu_{\eta}+\frac{B_{0}}{f_{0}^{2}}\left(4\left(\hat{m}+m_{\mathrm{s}}\right) L_{5}+8\left(2 \hat{m}+m_{\mathrm{s}}\right) L_{4}\right)\right\}
\end{aligned}
$$

where $\hat{m}=\left(m_{\mathrm{u}}+m_{\mathrm{s}}\right) / 2$ and $L_{4,5,6,8}$ are the low energy constants, and $\mu_{\mathrm{PS}}$ is the chiral logarithm defined by

$$
\mu_{\mathrm{PS}}=\frac{1}{32 \pi^{2}} \frac{m_{\mathrm{PS}}^{2}}{f_{0}^{2}} \ln \left(\frac{m_{\mathrm{PS}}^{2}}{\mu^{2}}\right)
$$

with $\mu$ the renormalization scale. There are six unknown low energy constants $B_{0}, f_{0}, L_{4,5,6,8}$ in the expressions above. The low energy constants are scale-dependent so as to cancel that of the chiral logarithm (3.5). We determine these parameters by making a simultaneous fit for $m_{\pi}^{2}, m_{K}^{2}, f_{\pi}$ and $f_{K}$.

We also consider the contributions of the finite size effects based on ChPT. At the one-loop level the finite size effect defined by $R_{X}=(X(L)-X(\infty)) / X(\infty)$ for $X=m_{\pi}, m_{K}, f_{\pi}, f_{K}$ is given by [11]:

$$
\begin{aligned}
R_{m_{\pi}} & =\frac{1}{4} \xi_{\pi} \tilde{g}_{1}\left(\lambda_{\pi}\right)-\frac{1}{12} \xi_{\eta} \tilde{g}_{1}\left(\lambda_{\eta}\right), \\
R_{m_{K}} & =\frac{1}{6} \xi_{\eta} \tilde{g}_{1}\left(\lambda_{\eta}\right), \\
R_{f_{\pi}} & =-\xi_{\pi} \tilde{g}_{1}\left(\lambda_{\pi}\right)-\frac{1}{2} \xi_{K} \tilde{g}_{1}\left(\lambda_{K}\right), \\
R_{f_{K}} & =-\frac{3}{8} \xi_{\pi} \tilde{g}_{1}\left(\lambda_{\pi}\right)-\frac{3}{4} \xi_{K} \tilde{g}_{1}\left(\lambda_{K}\right)-\frac{3}{8} \xi_{\eta} \tilde{g}_{1}\left(\lambda_{\eta}\right)
\end{aligned}
$$

with

$$
\xi_{\mathrm{PS}} \equiv \frac{m_{\mathrm{PS}}^{2}}{\left(4 \pi f_{\pi}\right)^{2}}, \quad \lambda_{\mathrm{PS}} \equiv m_{\mathrm{PS}} L, \quad \tilde{g}_{1}(x)=\sum_{n=1}^{\infty} \frac{4 m(n)}{\sqrt{n} x} K_{1}(\sqrt{n} x),
$$

where $K_{1}$ is the Bessel function of the second kind and $m(n)$ denotes the multiplicities in the expression of $n=n_{x}^{2}+n_{y}^{2}+n_{z}^{2}$. With the use of these formulae we estimate the possible finite size effects in our results.

\subsection{ChPT fits}

We apply the ChPT formulae (3.1)-(3.4) to our results at four points $\left(\kappa_{\mathrm{ud}}, \kappa_{\mathrm{s}}\right)=(0.13781$, $0.13640),(0.13770,0.13640),(0.13754,0.13640),(0.13754,0.13660)$. For these points, the $\rho$ meson mass satisfies the condition $m_{\rho}>2 m_{\pi}$. The measured AWI qurak masses are used for $\hat{m}$ and $m_{\mathrm{s}}$ in eqs.(3.1)-(3.4). The heaviest pion mass at $\left(\kappa_{\mathrm{ud}}, \kappa_{\mathrm{s}}\right)=(0.13754,0.13640)$ is about $430 \mathrm{MeV}$ with the use of the cutoff determined below. The fit results are shown in Fig. 2, where the black solid lines are drawn with $\kappa_{\mathrm{s}}$ fixed at 0.13640 and the black dotted lines are for $\kappa_{\mathrm{s}}=0.13660$. The red solid symbols represent the extrapolated values at the physical point whose determination is 


\begin{tabular}{ccccc}
\hline$L_{i}\left(\mu=m_{\eta}\right)$ & PACS-CS & PACS-CS with FSE & exp. value[12] & MILC[13] \\
\hline \hline$L_{4}$ & $0.25(11)$ & $0.23(12)$ & $0.27 \pm 0.8$ & $0.1(2)(2)$ \\
$L_{5}$ & $2.28(13)$ & $2.29(14)$ & $2.28 \pm 0.1$ & $2.0(3)(2)$ \\
$2 L_{6}-L_{4}$ & $0.16(4)$ & $0.16(4)$ & $0 \pm 1.0$ & $0.5(1)(2)$ \\
$2 L_{8}-L 5$ & $-0.59(5)$ & $-0.60(5)$ & $0.18 \pm 0.5$ & $-0.1(1)(1)$ \\
\hline$\chi^{2}$ d.o.f. & $2.1(1.4)$ & $2.1(1.4)$ & & \\
\hline
\end{tabular}

Table 2: Results for the low energy constants together with the phenomenological estimates and the MILC results.

explained in Sec. 3.4. The heaviest point at $\left(\kappa_{\mathrm{ud}}, \kappa_{\mathrm{s}}\right)=(0.13754,0.13640)$ is not well described by ChPT both for $\left(a m_{\pi}\right)^{2} /\left(a m_{\mathrm{ud}}^{\mathrm{AWI}}\right)$ and $f_{K} / f_{\pi}$, and $\chi^{2} /$ d.o.f. is rather large (see Table 2$)$.

The results for the low energy constants are presented in Table 2 where the phenomenological values with the experimental inputs[12] and the MILC results[13] are given for comparison. The renormalization scale is chosen to be $m_{\eta}=0.547 \mathrm{GeV}$. For $L_{4}$ and $L_{5}$ governing the behavior of $f_{\pi}, f_{K}$, our results show good agreement with both the phenomenological estimates and the MILC results. On the other hand, some discrepancies are observed between three results for $2 L_{6}-L_{4}$ and $2 L_{8}-L_{5}$ which enter into the ChPT formulae for $m_{\pi}^{2}$ and $m_{K}^{2}$.

In Fig. 2 we also draw the ChPT fit results incorporating the finite size effects. The blue solid lines are drawn for $\kappa_{\mathrm{s}}=0.13640$ and the blue dotted ones for $\kappa_{\mathrm{s}}=0.13660$. The fit curves with and without the finite size effects are almost degenerate for $a m_{\mathrm{ud}}^{\mathrm{AWI}}>0.003$, but we find a sizable difference at the physical point comparing the red open and solid symbols. This feature is understood by Fig. 3 where we plot the magnitude of $R_{X}$ for $X=m_{\pi}, m_{K}, f_{\pi}, f_{K}$ with $L=2.8 \mathrm{fm}$ as a function of $m_{\pi}$ (we note that $R_{m_{\mathrm{PS}}}>0$ and $R_{f_{\mathrm{PS}}}<0$ ); the finite size effects are less than $2 \%$ for $m_{\mathrm{PS}}$ and $f_{\mathrm{PS}}$ at our simulation points. This is true even at the physical point except for $f_{\pi}$ which decreases by $4 \%$.
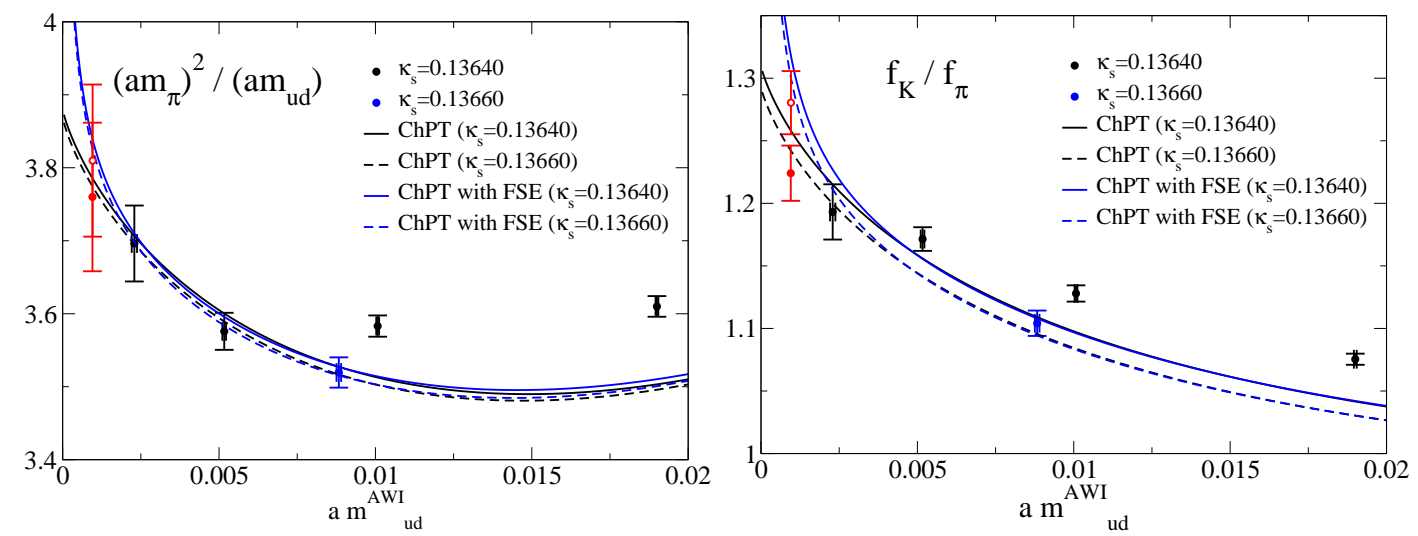

Figure 2: Fit results for $\left(a m_{\pi}\right)^{2} /\left(a m_{\mathrm{ud}}^{\mathrm{AWI}}\right)$ (left) and $f_{K} / f_{\pi}$ (right). Red solid (open) symbols denote the extrapolated values at the physical point by the ChPT formulae without (with) the finite size effects.

\subsection{Physical point and light hadron spectrum}

In order to determine the up-down and the strange quark masses and the lattice cutoff we need 


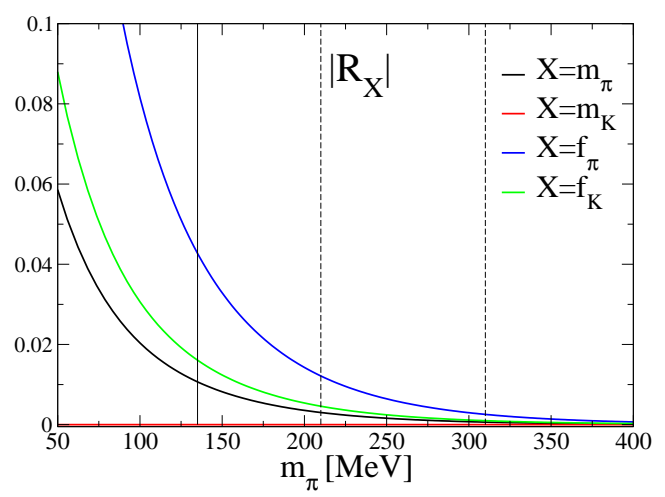

Figure 3: $\left|R_{X}\right|$ for $X=m_{\pi}, m_{K}, f_{\pi}, f_{K}$ with $L=2.8 \mathrm{fm}$ as a function of $m_{\pi}$. Solid vertical line denotes the physical point and the dotted ones are for our simulation points.

three physical inputs. We try the following two cases: $m_{\pi}, m_{K}, m_{\Omega}$ and $m_{\pi}, m_{K}, m_{\phi}$. The choice of $m_{\Omega}$ has theoretical and practical advantages: the $\Omega$ baryon is stable in the strong interactions and its mass, being composed of three strange quarks, is determined with good precision with small finite size effects. We also choose $m_{\phi}$ for comparison. We employ the NLO ChPT formulae for the chiral extrapolations of $m_{\pi}, m_{K}, f_{\pi}$ and $f_{K}$. A simple linear formula $m_{\mathrm{had}}=a+b \cdot m_{\mathrm{ud}}^{\mathrm{AWI}}+c \cdot m_{\mathrm{s}}^{\mathrm{AWI}}$ is used for the other hadron masses, employing data in the same range $\kappa_{\mathrm{ud}} \geq 0.13754$ as for pseudoscalar mesons. The results for the quark masses and the lattice cutoff are given by

$$
\begin{array}{llll}
a^{-1}=2.256(81) \mathrm{GeV}, & m_{u d}^{\overline{\mathrm{MS}}}=2.37(11) \mathrm{MeV}, & m_{s}^{\overline{\mathrm{MS}}}=69.1(25) \mathrm{MeV}, & m_{\Omega}-\text { input } \\
a^{-1}=2.248(76) \mathrm{GeV}, & m_{u d}^{\overline{\mathrm{MS}}}=2.38(11) \mathrm{MeV}, & m_{s}^{\overline{\mathrm{MS}}}=69.4(25) \mathrm{MeV}, & m_{\phi}-\text { input }
\end{array}
$$

where the errors are statistical. The two sets of results are consistent within the error. The quark masses are smaller than the recent estimates in the literature. We note, however, that we employed the perturbative renormalization factors to one-loop level which may contain a sizable uncertainty. A non-perutrbative calculation of the renormalization factor is in progress using the Schrödinger functional scheme.

Using the physical quark masses and the cutoff determined above, we obtain predictions for the pseudoscalar meson decay constants at the physical point:

$$
\begin{aligned}
& f_{\pi}=144(6) \mathrm{MeV}, \quad f_{K}=175(6) \mathrm{MeV}, f_{K} / f_{\pi}=1.219(22), \quad m_{\Omega} \text {-input } \\
& f_{\pi}=143(6) \mathrm{MeV}, f_{K}=175(5) \mathrm{MeV}, f_{K} / f_{\pi}=1.219(21), \quad m_{\phi} \text {-input, }
\end{aligned}
$$

to be compared with the experimantal values $f_{\pi}=130.7 \mathrm{MeV}, f_{K}=159.8 \mathrm{MeV}, f_{K} / f_{\pi}=1.223$. A $10 \%$ discrepancy in the magnitude of $f_{\pi}$ and $f_{K}$ might be due to use of one-loop perturbative $Z_{A}$ since the ratio shows a good agreement. A non-nonperturbative calculation of $Z_{A}$ and $Z_{m}=Z_{A} / Z_{P}$ is also in progress.

In Fig. 4 we compare the light hadron spectrum extrapolated to the physical point with the experiment. The results for the $\Omega$-input and the $\phi$-input are consistent with each other, and both are in agreement with the experiment albeit errors are still not small for some of the hadrons. We find this to be encouraging. Further work is of course needed since cutoff errors of $O\left(\left(a \Lambda_{\mathrm{QCD}}\right)^{2}\right)$ are present in our results. 


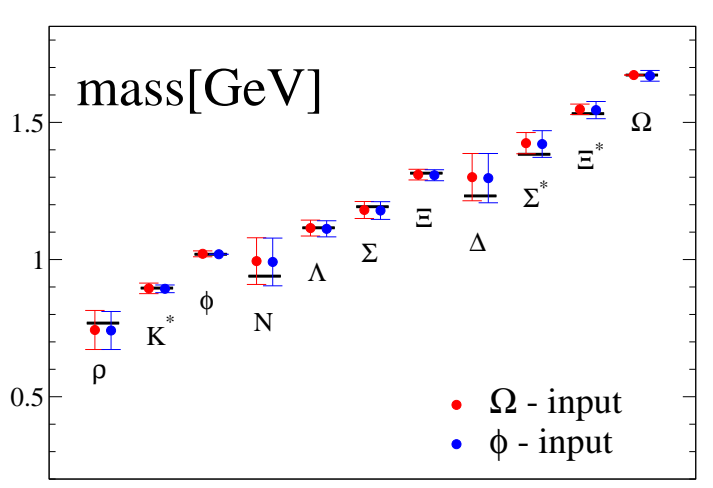

Figure 4: Light hadron spectrum extrapolated to the physical point with $\Omega$-input (red) and $\phi$-input (blue). Horizontal bars denote the experimental values.

\section{Acknowledgment}

This work is supported in part by Grants-in-Aid for Scientific Research from the Ministry of Education, Culture, Sports, Science and Technology (Nos. 13135204, 15540251, 17340066, 17540259, 18104005, 18540250, 18740139).

\section{References}

[1] PACS-CS Collaboration, S. Aoki et al., PoS (LAT2005) 111; A. Ukawa et al., PoS (LAT2006) 039.

[2] PACS-CS Collaboration, Y. Kuramashi et al., PoS(LAT2006) 029.

[3] PACS-CS Collaboration, Y. Kuramashi et al., a plenary talk in LAT2007.

[4] PACS-CS Collaboration, N. Ukita et al., PoS(LAT2007) 138.

[5] M. Lüscher, JHEP 0305 (2003) 052; Comput. Phys. Commun. 165 (2005) 199.

[6] CP-PACS Collaboration, S. Aoki et al., Phys. Rev. D73 (2006) 034501.

[7] T. Kaneko et al., JHEP 0704 (2007) 092.

[8] S. Aoki et al., Phys. Rev. D58 (1998) 074505.

[9] Y. Taniguchi and A. Ukawa, Phys. Rev. D58 (1998) 114503.

[10] J. Gasser and H. Leutwyler, Ann of Phys. 158 (1984) 142; Nucl. Phys. B250 (1985) 465.

[11] G. Colangelo, S. Dürr and C. Haefeli, Nucl. Phys. B 721 (2005) 136.

[12] G. Amorós, J. Bijnens and P. Talavera, Nucl. Phys. B602 (2001) 87.

[13] C. Bernard et al., arXiv:hep-lat/0611024. 